\title{
On the number of abelian groups of a given order (supplement)
}

by

\author{
Hong-QUAN LiU (Harbin)
}

To the days I lived in Manhattan, New York (90.9-91.6)

1. Introduction. The aim of this paper is to supply a still better result for the problem considered in [2]. Let $A(x)$ denote the number of distinct abelian groups (up to isomorphism) of orders not exceeding $x$. We shall prove

Theorem 1. For any $\varepsilon>0$,

$$
A(x)=C_{1} x+C_{2} x^{1 / 2}+C_{3} x^{1 / 3}+O\left(x^{50 / 199+\varepsilon}\right),
$$

where $C_{1}, C_{2}$ and $C_{3}$ are constants given on page 261 of [2].

Note that $50 / 199=0.25125 \ldots$, thus improving our previous exponent $40 / 159=0.25157 \ldots$ obtained in [2].

To prove Theorem 1, we shall proceed along the line of approach presented in [2]. The new tool here is an improved version of a result about enumerating certain lattice points due to E. Fouvry and H. Iwaniec (Proposition 2 of [1], which was listed as Lemma 6 in [2]).

2. A result about enumerating certain lattice points. In this section we prove the following improved version of Proposition 2 of [1].

Theorem 2. Let $Q \geq 1, m \sim M, q \sim Q$, let $\alpha(\neq 0,1)$ be a real number, $t(m, q)=(m+q)^{\alpha}-(m-q)^{\alpha}, T=M^{\alpha-1} Q$, and let $B(M, Q, \Delta)$ be the number of lattice points $\left(m, m_{1}, q, q_{1}\right)$ such that

$$
\left|t(m, q)-t\left(m_{1}, q_{1}\right)\right|<\Delta T .
$$

If $Q<\varepsilon M^{3 / 4}$, where $\varepsilon$ is a sufficiently small positive number, we have

$$
B(M, Q, \Delta) \ll\left(M Q+\Delta M^{2} Q^{2}+Q^{8 / 3}\right)(\log 2 M)^{4},
$$

where the $\ll$ constant depends at most on $\alpha$ and $\varepsilon$. 
It is obvious that Theorem 2 follows from the next two lemmas.

Lemma 1. Let $B_{1}\left(M, Q, \Delta_{1}\right)$ be the number of lattice points $\left(m_{1}, q, q_{1}\right)$ such that $m_{1} \sim M, q, q_{1} \sim Q$ and

$$
\left\|\left(\frac{q_{1}}{q}\right)^{\beta} m_{1}+d_{1} m_{1}^{-1} f\left(q, q_{1}\right)+m_{1}^{-3} g\left(q, q_{1}\right)\right\| \leq \varepsilon^{-1} \Delta_{1},
$$

where

$$
\begin{gathered}
\|x\|=\min _{n \in \mathbb{Z}}|n-x|, \quad \Delta_{1}=\Delta M+Q^{6} M^{-5}, \quad \beta=\frac{1}{\alpha-1}, \\
f\left(q, q_{1}\right)=q^{2}\left(\frac{q}{q_{1}}\right)^{\beta}-q_{1}^{2}\left(\frac{q_{1}}{q}\right)^{\beta}, \\
g\left(q, q_{1}\right)=d_{2}\left(q^{4}\left(\frac{q}{q_{1}}\right)^{3 \beta}-q_{1}^{4}\left(\frac{q_{1}}{q}\right)^{\beta}\right)-d_{1}^{2} q^{2}\left(q_{1}^{2}\left(\frac{q}{q_{1}}\right)^{\beta}-q^{2}\left(\frac{q}{q_{1}}\right)^{3 \beta}\right),
\end{gathered}
$$

and $d_{1}, d_{2}$ are the constants given by the Taylor expansion

$$
\left(\frac{(1+u)^{\alpha}-(1-u)^{\alpha}}{2 \alpha u}\right)^{\beta}=1+d_{1} u^{2}+d_{2} u^{4}+\ldots, \quad 0<u<1 .
$$

Then, for $Q<M^{5 / 6-\varepsilon}$,

$$
B(M, Q, \Delta) \ll B_{1}\left(M, Q, \Delta_{1}\right) .
$$

Proof. We assume that $\Delta M$ is small, for otherwise Theorem 2 follows immediately from the inequality

$$
\left|t(m, q)-t\left(m_{1}, q_{1}\right)\right|<\Delta T .
$$

From (1) it is easy to see that the Taylor expansion implies

(2) $\quad m\left(1+d_{1}\left(\frac{q}{m}\right)^{2}+d_{2}\left(\frac{q}{m}\right)^{4}\right)$

$$
-\left(\frac{q_{1}}{q}\right)^{\beta} m_{1}\left(1+d_{1}\left(\frac{q_{1}}{m_{1}}\right)^{2}+d_{2}\left(\frac{q_{1}}{m_{1}}\right)^{4}\right) \ll \Delta_{1} .
$$

From (2) we get

$$
m=\left(\frac{q_{1}}{q}\right)^{\beta} m_{1}\left(1+O\left(\Delta+Q^{2} M^{-2}\right)\right),
$$

and

(4) $m-\left(\frac{q_{1}}{q}\right)^{\beta} m_{1}+d_{1}\left(q^{2} m^{-1}-q_{1}^{2} m_{1}^{-1}\left(\frac{q_{1}}{q}\right)^{\beta}\right)=O\left(\Delta M+Q^{4} M^{-3}\right)$. 
By substituting (3) into (4), we get a more precise expansion

(5) $m=m_{1}\left(\frac{q_{1}}{q}\right)^{\beta}+d_{1} m_{1}^{-1}\left(q_{1}^{2}\left(\frac{q_{1}}{q}\right)^{\beta}-q^{2}\left(\frac{q}{q_{1}}\right)^{\beta}\right)+O\left(\Delta M+Q^{4} M^{-3}\right)$.

We now use (3) to expand $d_{2} q^{4} m^{-3}$ and use (5) to expand $d_{1} q^{2} m^{-1}$, thereby obtaining, in view of $(2)$, the estimate

$$
m-\left(\frac{q_{1}}{q}\right)^{\beta} m_{1}+d_{1} m_{1}^{-1} f\left(q, q_{1}\right)+m_{1}^{-3} g\left(q, q_{1}\right) \ll \Delta_{1} .
$$

Lemma 1 follows from (6) and the fact that $\Delta_{1}$ is small.

Lemma 2. Let $B_{1}\left(M, Q, \Delta_{1}\right)$ be defined in Lemma 1 and $Q<\varepsilon M^{3 / 4}$. Then

$$
B_{1}\left(M, Q, \Delta_{1}\right) \ll\left(M Q+\Delta M^{2} Q^{2}+Q^{8 / 3}\right)(\log 2 M)^{4} .
$$

Proof. Let $\Delta_{2}=\Delta M+M^{-1} Q^{2 / 3}$. Clearly,

$$
B_{1}\left(M, Q, \Delta_{1}\right) \leq B_{1}\left(M, Q, \Delta_{2}\right) \text {. }
$$

For fixed $\left(q, q_{1}\right)$, the number of lattice points counted in $B_{1}\left(M, Q, \Delta_{2}\right)$ is (with $S=\varepsilon\left(4 \Delta_{2}\right)^{-1}$ )

$$
\ll S^{-1} \sum_{1 \leq s \leq S}\left|\sum_{m \sim M} e\left(A s m+B s m^{-1}+C s m^{-3}\right)\right|+\Delta_{2} M,
$$

by virtue of the identity

$$
\sum_{|s|<S}\left(1-\frac{|s|}{S}\right) e(s x)=\frac{1-\{S\}}{S}\left(\frac{\sin \pi x[S]}{\sin \pi x}\right)^{2}+\frac{\{S\}}{S}\left(\frac{\sin \pi x[S+1]}{\sin \pi x}\right)^{2}
$$

in (7), $A, B$ and $C$ are given by

$$
A=\left(\frac{q_{1}}{q}\right)^{\beta}, \quad B=d_{1} f\left(q, q_{1}\right), \quad C=g\left(q, q_{1}\right) .
$$

Under our assumption, the innermost sum in (7) is

$$
\int_{M}^{2 M} e\left( \pm\|A s\| \xi+B s \xi^{-1}+C s \xi^{-3}\right) d \xi+O(1)=I+O(1), \quad \text { say, }
$$

by using the truncated Poisson's summation formula.

If $\|A s\| \geq 3 s|B| M^{-2}$, then by partial integration,

$$
I \ll\|s A\|^{-1}
$$

and if $\|A s\|<3 s|B| M^{-2}$, then we apply the well-known second derivative estimate to get

$$
I \ll(s|B|)^{-1 / 2} M^{3 / 2} \quad \text { for } B \neq 0,
$$


where we have used the fact that $|C| \ll|B| Q^{2}$. From (7)-(10) we conclude that

$$
B_{1}\left(M, Q, \Delta_{2}\right) \ll \Delta_{2} M Q^{2}+E_{1}\left(M, Q, \Delta_{2}\right)+E_{2}\left(M, Q, \Delta_{2}\right),
$$

where

$$
\begin{aligned}
& E_{1}\left(M, Q, \Delta_{2}\right)=\Delta_{2} \sum_{1 \leq s \leq S} \sum_{\substack{1, q_{1} \sim Q\\
}} \min (M, 1 /\|A s\|), \\
& E_{2}\left(M, Q, \Delta_{2}\right)=\Delta_{2} \sum_{\substack{1 \leq s \leq S \\
\|A s\|<3 s|B| M_{1} \sim Q}} \sum_{\substack{-2 \\
\| A n}} \min \left(M,(s|B|)^{-1 / 2} M^{3 / 2}\right) .
\end{aligned}
$$

$E_{i}\left(M, Q, \Delta_{2}\right)(i=1,2)$ can be estimated just as $D_{i}(M, Q, \Delta)$ on page 320 of [1], and we have

$$
\begin{gathered}
E_{1}\left(M, Q, \Delta_{2}\right) \ll M Q(\log 2 M)^{3}, \\
E_{2}\left(M, Q, \Delta_{2}\right) \ll\left(M Q+\left(\Delta_{2} M\right)^{-1 / 2} Q^{3}\right)(\log 2 M)^{4} .
\end{gathered}
$$

Lemma 2 follows from (11)-(13).

3. A bound for a kind of triple exponential sums. By means of Theorem 2, we can sharpen Lemma A of [2] as follows. We have

Theorem 3. Let $H \geq 1, X \geq 1, Y \geq 1000$; let $\alpha, \beta$ and $\gamma$ be real numbers such that $\alpha \gamma(\gamma-1)(\beta-1) \neq 0$, and $A>C(\alpha, \beta, \gamma)>0, f(h, x, y)=$ $A h^{\alpha} x^{\beta} y^{\gamma}$. Define

$$
S(H, X, Y)=\sum_{(h, x, y) \in D} C_{1}(h, x) C_{2}(y) e(f(h, x, y)),
$$

where $D$ is a region contained in the rectangle

$$
\{(h, x, y) \mid h \sim H, x \sim X, y \sim Y\}
$$

such that for any fixed pair $\left(h_{0}, x_{0}\right)$, the intersection $D \cap\left\{\left(h_{0}, x_{0}, y\right) \mid y \sim Y\right\}$ has at most $O(1)$ segments. Also, suppose $\left|C_{1}(h, x)\right| \leq 1,\left|C_{2}(y)\right| \leq 1$, $F=A H^{\alpha} X^{\beta} Y^{\gamma} \gg Y$. Then

$$
\begin{aligned}
L^{-3} S(H, X, Y) \ll & \sqrt[22]{(H X)^{19} Y^{13} F^{3}}+H X Y^{5 / 8}\left(1+Y^{7} F^{-4}\right)^{1 / 16} \\
& +\sqrt[32]{(H X)^{29} Y^{28} F^{-2} M^{5}}+\sqrt[4]{(H X)^{3} Y^{4} M} \\
\equiv & E_{1},
\end{aligned}
$$

where $L=\log (A H X Y+2), M=\max \left(1, F Y^{-2}\right)$.

Proof. We have

$$
S(H, X, Y) \ll \sum_{h \sim H} \sum_{x \sim X}\left|\sum_{y \in I(h, x)} C_{2}(y) e(f(h, x, y))\right|,
$$


where $I(h, x)$ is some subinterval of $(Y, 2 Y]$. From Lemma 1 of [2], we get

$$
L^{-1} S(H, X, Y) \ll \sum_{h \sim H} \sum_{x \sim X}\left|\sum_{y \sim Y} C(y, \theta) e(f(h, x, y))\right|,
$$

where $C(y, \theta)=C_{2}(y) e(\theta y)$ for some real number $\theta$ ( $\theta$ is independent of $h$, $x$, and $y)$. We consider the expression

$$
\begin{aligned}
R(q)= & (H X Y)^{2} q^{-1}+(H X)^{2}\left(Y^{5} F^{-1} M q^{-1}\right)^{1 / 2} \\
& +\sqrt[6]{(H X)^{9} Y^{3} F^{3} q^{5}}+(H X)^{2} Y q^{1 / 3}+\sqrt{(H X)^{3} Y^{4} M}
\end{aligned}
$$

By Lemma 2 of [2], we can choose a $Q \in\left(0, \varepsilon Y^{3 / 4}\right]$ such that

$$
\begin{aligned}
R(Q) \ll & \sqrt[11]{(H X)^{19} Y^{13} F^{3}}+(H X)^{2} Y^{5 / 4}+(H X)^{2}\left(F^{-4} M^{4} Y^{17}\right)^{1 / 8} \\
& +(H X)^{2}\left(Y^{8} F^{-1} M\right)^{1 / 5}+\sqrt[16]{(H X)^{29} Y^{28} F^{-2} M^{5}} \\
& +\sqrt{(H X)^{3} Y^{4} M} \ll E_{1}^{2}
\end{aligned}
$$

(see (14)). If $Q \leq 100$, then we trivially have

$$
L^{-1} S(H, X, Y) \ll H X Y Q^{-1 / 2} \ll \sqrt{R(Q)} \ll E_{1} .
$$

Now we assume that $Q>100$. By Cauchy's inequality and Lemma 3 of [2], we get

$$
L^{-3}|S(H, X, Y)|^{2} \ll(H X Y)^{2} Q^{-1}+(H X Y) Q^{-1}\left|S_{1}\right|
$$

where

$$
\begin{gathered}
S_{1}=\sum_{(q, y, h, x) \in D_{1}} C(y+q, \theta) \overline{C(y-q, \theta)} e\left(A h^{\alpha} x^{\beta} t(y, q)\right), \\
t(y, q)=(y+q)^{\gamma}-(y-q)^{\gamma}, \\
D_{1}=D_{1}\left(Q_{1}\right)=\left\{(q, y, h, x) \mid y+q, y-q \sim Y, q \sim Q_{1}, h \sim H, x \sim X\right\}
\end{gathered}
$$

for some $Q_{1}$ with $1 \leq 2 Q_{1} \leq Q / 2$. By Lemma 4 of [2] we have (note that $F \gg Y$ by our assumption)

$$
\left|S_{1}\right|^{2} \ll F Y^{-1} Q_{1} A_{1} A_{2},
$$

where $A_{1}$ is the number of lattice points $\left(h, x, h_{1}, x_{1}\right)$ such that

$$
\left|h^{\alpha} x^{\beta}-h_{1}^{\alpha} x_{1}^{\beta}\right| \ll A^{-1} Q_{1}^{-1} Y^{1-\gamma}
$$

with $h, h_{1} \sim H, x, x_{1} \sim X$, which is estimated by Lemma 5 of [2] as

$$
A_{1} \ll\left(H X+H^{2} X^{2} Y Q_{1}^{-1} F^{-1}\right) L^{2}
$$

and $A_{2}$ stands for the number of lattice points $\left(q, y, q_{1}, y_{1}\right)$ such that

$$
\left|t(y, q)-t\left(y_{1}, q_{1}\right)\right| \ll\left(A H^{\alpha} X^{\beta}\right)^{-1}
$$


with $Y / 2<y, y_{1}<3 Y, q, q_{1} \sim Q_{1}$. Recall that $Q_{1} \leq Q / 4<\varepsilon Y^{3 / 4}$. Theorem 2 gives (with $\Delta=Q_{1}^{-1} Y F^{-1}$ )

$$
A_{2} \ll\left(Q_{1} Y+Q_{1} Y^{3} F^{-1}+Q_{1}^{8 / 3}\right) L^{4} .
$$

From (17)-(20), we deduce that (see (15))

(21) $\quad L^{-6}|S(H, X, Y)|^{2} \ll(H X Y)^{2} Q^{-1}$

$+H X Y Q^{-1}\left(F H X Q\left(Q+H X Y F^{-1}\right)\left(1+Y^{2} F^{-1}+Q^{5 / 3} Y^{-1}\right)\right)^{1 / 2} \ll R(Q)$.

Theorem 3 follows from (21) and (16).

4. The proof of Theorem 1. Put

$$
\theta=50 / 199, \quad S_{1,2,3}=\sum_{\substack{m n \leq x^{1 / 3} \\ m>n}} \Psi\left(x m^{-2} n^{-3}\right), \quad \Psi(u)=u-[u]-1 / 2 .
$$

By Lemmas 7, 8 and Theorems 1, 2 of [2], to prove Theorem 1 it is sufficient to establish the following lemma.

LEMMA B.

$$
S_{1,2,3} \ll x^{\theta+\varepsilon} .
$$

Obviously, we have

$$
S_{1,2,3}=\sum_{(M, N)} S_{1,2,3}(M, N)+O\left(x^{\theta+\varepsilon}\right),
$$

where $M$ and $N$ run through the sequences $\left\{2^{-j} x^{1 / 3} \mid j=0,1, \ldots\right\}$ and $\left\{2^{-k} x^{1 / 3} \mid k=0,1, \ldots\right\}$ respectively, such that

$$
M N \geq x^{\theta}, \quad 2 M \geq N, \quad M N \leq x^{1 / 3},
$$

and

$$
S_{1,2,3}(M, N)=\sum_{(m, n) \in D} \Psi\left(x m^{-2} n^{-3}\right),
$$

$$
D=D(M, N)=\left\{(m, n) \mid m \sim M, n \sim N, m n \leq x^{1 / 3}, m>n\right\} .
$$

By means of the standard expansion for the function $\Psi(\cdot)$, we get, for any parameter $K, K \in[100, M N]$, the inequality

$$
\begin{aligned}
& (\log K)^{-1} S_{1,2,3}(M, N) \\
& \ll M N K^{-1}+\sum_{1 \leq h \leq K^{2}} \min \left(\frac{1}{h}, \frac{K}{h^{2}}\right)\left|\sum_{(m, n) \in D} e(f(h, m, n))\right|,
\end{aligned}
$$

where $f(h, m, n)=h x m^{-2} n^{-3}$. Thus, for some $H \in\left[1, K^{2}\right]$, we have

$$
x^{-\varepsilon} S_{1,2,3}(M, N) \ll M N K^{-1}+\min (1, K / H) \Phi_{1,2,3}(H, M, N),
$$


where

$$
\Phi_{1,2,3}(H, M, N)=H^{-1} \sum_{h \sim H}\left|\sum_{(m, n) \in D} e(f(h, m, n))\right|
$$

(we have adopted the notations on pp. 266-267 of [2]). We now use our Theorem 3 three times to estimate the sum $S_{1,2,3}(M, N)$. Lemma B will then be proved by invoking (49) of [2].

LEMMA 3.

$$
\begin{aligned}
x^{-\varepsilon} S_{1,2,3}(M, N) \ll & \sqrt[30]{x^{11} M^{-11} N^{-12}}+\sqrt[12]{x^{4} M^{-4} N^{-3}} \\
& +\sqrt[45]{x^{16} M^{-16} N^{-17}}+\sqrt[5]{x^{2} M^{-2} N^{-3}}+x^{1 / 4} \equiv E_{2} .
\end{aligned}
$$

Proof. We use Lemma 10 of [2] to the summation over $m$, and obtain, in view of (23),

$$
\begin{aligned}
& \sum_{(m, n) \in D} e(f(h, m, n)) \\
&=c_{1}(h x)^{1 / 6} \sum_{(n, u) \in D_{1}}\left(n^{3} u^{4}\right)^{-1 / 6} e(g(h, n, u))+O\left(x^{1 / 4}\right),
\end{aligned}
$$

where

$$
\begin{gathered}
g(h, n, u)=c_{2}\left(x h n^{-3} u^{2}\right)^{1 / 3}, \\
D_{1}=\left\{(n, u) \mid u n^{6} \leq c_{3} h x, h \leq c_{4} u, n \sim N, c_{5} \leq h x /\left(n^{3} u M^{3}\right) \leq c_{6}\right\},
\end{gathered}
$$

with $c_{i}(1 \leq i \leq 6)$ being some absolute constants. From (26) and (27), we find that

$$
\begin{aligned}
& x^{-\varepsilon / 2} \Phi_{1,2,3}(H, M, N) \\
& \quad \ll M\left(H^{3} G\right)^{-1 / 2} \sum_{h \sim H}\left|\sum_{(n, u) \in D_{1}} C(n) \widetilde{C}(u) e(g(h, n, u))\right|+x^{1 / 4},
\end{aligned}
$$

where $|C(n)| \leq 1,|\widetilde{C}(u)| \leq 1$, and $G=x M^{-2} N^{-3}$. We apply Theorem 3 with $(H, X, Y) \simeq(H, G H / M, N)$ to get (note that $(n, u) \in D_{1}$ implies $u \simeq G H / M)$

$$
\begin{aligned}
x^{-\varepsilon / 2} & \sum_{h \sim H}\left|\sum_{(n, u) \in D_{1}} C(n) \widetilde{C}(u) e(g(h, n, u))\right| \\
\ll & \sqrt[22]{H^{41} G^{22} M^{-19} N^{13}}+H^{2} G M^{-1} N^{5 / 8}+\sqrt[16]{H^{28} G^{12} M^{-16} N^{11}} \\
& +\sqrt[32]{H^{56} G^{27} M^{-29} N^{28}}+\sqrt[32]{H^{61} G^{32} M^{-29} N^{18}} \\
& +\sqrt[4]{H^{6} G^{3} M^{-3} N^{4}}+\sqrt[4]{H^{7} G^{4} M^{-3} N^{2}} .
\end{aligned}
$$


From (25), (26), (28) and (29), we obtain

$$
\begin{aligned}
x^{-\varepsilon} S_{1,2,3}(M, N) \ll & M N K^{-1}+\sqrt[22]{K^{8} x^{11} M^{-19} N^{-20}} \\
& +\sqrt[8]{K^{4} x^{4} M^{-8} N^{-7}}+\sqrt[16]{K^{4} x^{4} M^{-8} N^{-1}} \\
& +\sqrt[32]{K^{8} x^{11} M^{-19} N^{-5}}+\sqrt[32]{K^{13} x^{16} M^{-29} N^{-30}} \\
& +\sqrt[4]{K x^{2} M^{-3} N^{-4}}+x^{1 / 4} \\
= & E_{2}(K)+x^{1 / 4}, \quad \text { say. }
\end{aligned}
$$

By Lemma 2 of [2], there exists a $K_{0} \in[0, M N]$ such that

$$
E_{2}\left(K_{0}\right) \ll E_{2} .
$$

If $K_{0} \geq 100$, we put $K=K_{0}$ in (30), and Lemma 3 follows from (30) and (31); if $K_{0}<100$, we trivially get

$$
S_{1,2,3}(M, N) \ll M N K_{0}^{-1} \ll E_{2}\left(K_{0}\right),
$$

and Lemma 3 follows from (32) and (31).

Lemma 4. For $K=M N x^{-\theta}, 1 \leq H \leq K^{2}$, we have

$$
\begin{aligned}
x^{-\varepsilon} \Phi_{1,2,3}(H, M, N) \ll & \sqrt[22]{x^{3} M^{7} N^{10}}+N M^{5 / 8}+\sqrt[16]{H^{-4} x^{-4} M^{25} N^{28}} \\
& +\sqrt[32]{H^{-5} x^{-2} M^{32} N^{35}}+\sqrt[32]{x^{3} M^{12} N^{20}} \\
& +\sqrt[4]{H^{-1} M^{4} N^{3}}+x^{1 / 4}
\end{aligned}
$$

Pr o of. Applying Theorem 3 to the sum $H \Phi_{1,2,3}(H, M, N)$ directly, with $(H, X, Y) \simeq(H, N, M)$, we get the required estimate.

LEMMA 5. For $K=M N x^{-\theta}, 1 \leq H \leq K^{2}$, we have

$$
\begin{gathered}
x^{-\varepsilon} \min (1, K / H) \Phi_{1,2,3}(H, M, N) \\
\ll \sqrt[22]{x^{5-2 \theta} M N^{6}}+\sqrt[8]{x^{1-\theta} M^{2} N^{6}}+\sqrt[32]{x^{5-2 \theta} M^{6} N^{16}}+\sqrt[32]{x^{4} M^{9} N^{16}} \\
+\sqrt[52]{x^{8} M^{12} N^{20}}+\min \left(\sqrt[4]{x^{2-\theta} M^{-3} N^{-2}}, \sqrt[22]{x^{3} M^{7} N^{10}}\right)+x^{\theta} .
\end{gathered}
$$

Pr o of. Applying Theorem 3 to the triple exponential sum of (28), with $(H, X, Y) \simeq(H, N, G H / M)$, we get

$$
\begin{aligned}
& x^{-\varepsilon / 2} \sum_{h \sim H}\left|\sum_{(n, u) \in D_{1}} C(n) \widetilde{C}(u) e(g(h, n, u))\right| \\
\ll & \sqrt[22]{H^{35} G^{16} M^{-13} N^{19}}+\sqrt[8]{H^{13} G^{5} M^{-5} N^{8}}+\sqrt[16]{H^{29} G^{13} M^{-17} N^{16}} \\
& +\sqrt[32]{H^{55} G^{26} M^{-28} N^{29}}+\sqrt[32]{H^{50} G^{21} M^{-18} N^{29}} \\
& +\sqrt[4]{H^{7} G^{4} M^{-4} N^{3}}+\sqrt[4]{H^{6} G^{3} M^{-2} N^{-3}}+x^{1 / 4} .
\end{aligned}
$$


From (28) and (33), we obtain

$$
\begin{aligned}
x^{-\varepsilon} \Phi_{1,2,3}(H, M, N) \ll & \sqrt[22]{H^{2} x^{5} M^{-1} N^{4}}+\sqrt[8]{H x M N^{5}}+\sqrt[16]{H^{5} x^{5} M^{-11} N} \\
& +\sqrt[32]{H^{7} x^{10} M^{-16} N^{-1}}+\sqrt[32]{H^{2} x^{5} M^{4} N^{14}} \\
& +\sqrt[4]{H x^{2} M^{-4} N^{-3}}+x^{1 / 4}
\end{aligned}
$$

which, in conjunction with Lemma 4 and (23), gives

$$
\begin{aligned}
x^{-\varepsilon} \min (1, K / H) & \Phi_{1,2,3}(H, M, N) \\
\ll & \sqrt[22]{x^{5-2 \theta} M N^{6}}+\sqrt[8]{x^{1-\theta} M^{2} N^{6}}+\sqrt[32]{x^{5-2 \theta} M^{6} N^{16}} \\
& +\min \left(\sqrt[4]{x^{2} H M^{-4} N^{-3}}, \sqrt[16]{H^{-4} x^{-4} M^{25} N^{28}}\right) \\
& +\min \left(\sqrt[4]{x^{2} H M^{-4} N^{-3}}, \sqrt[32]{H^{-5} x^{-2} M^{32} N^{35}}\right) \\
& +\min \left(\sqrt[4]{x^{2} H M^{-4} N^{-3}}, \sqrt[4]{H^{-1} M^{4} N^{3}}\right) \\
& +\min \left(\sqrt[4]{x^{2-\theta} M^{-3} N^{-2}}, \sqrt[22]{x^{3} M^{7} N^{10}}\right) \\
& +\min \left(\sqrt[4]{x^{2-\theta} M^{-3} N^{-2}}, N M^{5 / 8}\right) \\
& +\min \left(\sqrt[4]{x^{2-\theta} M^{-3} N^{-2}}, \sqrt[32]{x^{3} M^{12} N^{20}}\right)+x^{\theta} .
\end{aligned}
$$

Obviously,

$$
\begin{gathered}
\min \left(\sqrt[4]{x^{2} H M^{-4} N^{-3}}, \sqrt[16]{H^{-4} x^{-4} M^{25} N^{28}}\right) \leq \sqrt[32]{x^{4} M^{9} N^{16}} \\
\min \left(\sqrt[4]{x^{2} H M^{-4} N^{-3}}, \sqrt[32]{H^{-5} x^{-2} M^{32} N^{35}}\right) \leq \sqrt[52]{x^{8} M^{12} N^{20}} \\
\min \left(\sqrt[4]{x^{2} H M^{-4} N^{-3}}, \sqrt[4]{H^{-1} M^{4} N^{3}}\right) \leq x^{1 / 4}
\end{gathered}
$$

and, in view of (23),

$$
\begin{aligned}
& \min \left(\sqrt[4]{x^{2-\theta} M^{-3} N^{-2}}, N M^{5 / 8}\right) \\
& \quad \ll \min \left(\sqrt[4]{x^{2-\theta} M^{-3} N^{-2}},\left(M^{3} N^{2}\right)^{13 / 40}\right) \leq x^{(26-13 \theta) / 92}<x^{\theta}, \\
& \min \left(\sqrt[4]{x^{2-\theta} M^{-3} N^{-2}}, \sqrt[32]{x^{3} M^{12} N^{20}}\right) \\
& \ll \min \left(\sqrt[4]{x^{2-\theta} M^{-3} N^{-2}}, \sqrt[32]{x^{3}\left(M^{3} N^{2}\right)^{32 / 5}}\right) \leq x^{(79-32 \theta) / 288}<x^{\theta} .
\end{aligned}
$$

From (34) to (39), Lemma 5 follows.

Proof of Lemma B. By (49) of [2], we have

$$
x^{-\varepsilon} S_{1,2,3}(M, N) \ll \sqrt[8]{x^{2} M N^{-1}} .
$$


By (25), Lemma 5 and (40), we get

$$
\begin{aligned}
x^{-\varepsilon} S_{1,2,3}(M, N) \ll & \sqrt[22]{x^{5-2 \theta} M N^{6}}+\sqrt[8]{x^{1-\theta} M^{2} N^{6}}+\sqrt[32]{x^{5-2 \theta} M^{6} N^{16}} \\
& +\sqrt[32]{x^{4} M^{9} N^{16}}+\sqrt[52]{x^{8} M^{12} N^{20}}+R_{1}(M, N)+x^{\theta}
\end{aligned}
$$

where

$$
\begin{aligned}
R_{1}(M, N) & =\min \left(\sqrt[22]{x^{3} M^{7} N^{10}}, \sqrt[4]{x^{2-\theta} M^{-3} N^{-2}}, \sqrt[8]{x^{2} M N^{-1}}\right) \\
& \leq\left(\sqrt[22]{x^{3} M^{7} N^{10}}\right)^{\alpha_{1}}\left(\sqrt[4]{x^{2-\theta} M^{-3} N^{-2}}\right)^{\beta_{1}}\left(\sqrt[8]{x^{2} M N^{-1}}\right)^{\gamma_{1}} \\
& =x^{(81-17 \theta) / 306}<x^{\theta},
\end{aligned}
$$

with $\left(\alpha_{1}, \beta_{1}, \gamma_{1}\right)=(110 / 306,68 / 306,128 / 306)$; thus

$$
\begin{aligned}
x^{-\varepsilon} S_{1,2,3}(M, N) \ll & \sqrt[22]{x^{5-2 \theta} M N^{6}}+\sqrt[8]{x^{1-\theta} M^{2} N^{6}} \\
& +\sqrt[32]{x^{5-2 \theta} M^{6} N^{16}}+\sqrt[32]{x^{4} M^{9} N^{16}} \\
& +\sqrt[52]{x^{8} M^{12} N^{20}}+x^{\theta} .
\end{aligned}
$$

If $M N \leq x^{0.3}$, then (41) gives

$$
x^{-\varepsilon} S_{1,2,3}(M, N) \ll \sqrt[22]{x^{5-2 \theta} M N^{6}}+x^{\theta} .
$$

From Lemma 3, (40) and (42), we deduce that

$$
x^{-\varepsilon} S_{1,2,3}(M, N) \ll \sum_{i=2}^{5} R_{i}(M, N)+x^{\theta},
$$

where

$$
\text { (44) } \begin{aligned}
R_{2}(M, N) & =\min \left(\sqrt[30]{x^{11} M^{-11} N^{-12}}, \sqrt[22]{x^{5-2 \theta} M N^{6}}, \sqrt[8]{x^{2} M N^{-1}}\right) \\
& \leq\left(\sqrt[30]{x^{11} M^{-11} N^{-12}}\right)^{\alpha_{2}}\left(\sqrt[22]{x^{5-2 \theta} M N^{6}}\right)^{\beta_{2}}\left(\sqrt[8]{x^{2} M N^{-1}}\right)^{\gamma_{2}} \\
& =x^{(150-23 \theta) / 574}=x^{\theta}
\end{aligned}
$$

with $\left(\alpha_{2}, \beta_{2}, \gamma_{2}\right)=(105 / 574,253 / 574,216 / 574)$;

$$
\begin{aligned}
R_{3}(M, N) & =\min \left(\sqrt[12]{x^{4} M^{-4} N^{-3}}, \sqrt[22]{x^{5-2 \theta} M N^{6}}\right) \\
& \ll\left(\sqrt[12]{x^{4} M^{-4} N^{-3}}\right)^{12 / 34}\left(\sqrt[22]{x^{5-2 \theta} M^{4} N^{3}}\right)^{22 / 34} \\
& =x^{(9-2 \theta) / 34}<x^{\theta} ;
\end{aligned}
$$

(46) $R_{4}(M, N)=\min \left(\sqrt[45]{x^{16} M^{-16} N^{-17}}, \sqrt[22]{x^{5-2 \theta} M N^{6}}\right)$

$$
\begin{aligned}
& \ll\left(\sqrt[45]{x^{16} M^{-16} N^{-17}}\right)^{105 / 347}\left(\sqrt[22]{x^{5-2 \theta}\left(M^{16} N^{17}\right)^{7 / 33}}\right)^{242 / 347} \\
& =x^{(277-66 \theta) / 1041}<x^{\theta}
\end{aligned}
$$

(47) $R_{5}(M, N)$

$$
=\min \left(\sqrt[5]{x^{2} M^{-2} N^{-3}}, \sqrt[22]{x^{5-2 \theta} M N^{6}}, \sqrt[8]{x^{2} M N^{-1}}\right)
$$




$$
\begin{aligned}
& \leq\left(\sqrt[5]{x^{2} M^{-2} N^{-3}}\right)^{35 / 217}\left(\sqrt[22]{x^{5-2 \theta} M N^{6}}\right)^{110 / 217}\left(\sqrt[8]{x^{2} M N^{-1}}\right)^{72 / 217} \\
& =x^{(57-10 \theta) / 217}<x^{\theta}
\end{aligned}
$$

From (43) to (47), we have

$$
x^{-\varepsilon} S_{1,2,3}(M, N) \ll x^{\theta} .
$$

If $M N>x^{0.3}$, from Lemma 3 we find

$$
x^{-\varepsilon} S_{1,2,3}(M, N) \ll \sqrt[30]{x^{11} M^{-11} N^{-12}}+\sqrt[5]{x^{2} M^{-2} N^{-3}}+x^{\theta} .
$$

From (40), (41) and (49), we deduce that

$$
x^{-\varepsilon} S_{1,2,3}(M, N) \ll \sum_{i=6}^{15} R_{i}(M, N)+x^{\theta},
$$

where, by (44) and (47),

$$
\begin{aligned}
R_{6}(M, N) & =R_{2}(M, N) \leq x^{\theta}, \quad R_{7}(M, N)=R_{5}(M, N)<x^{\theta} \\
R_{8}(M, N) & =\min \left(\sqrt[30]{x^{11} M^{-11} N^{-12}}, \sqrt[8]{x^{1-\theta} M^{2} N^{6}}\right) \\
\ll & \left(\sqrt[30]{x^{11} M^{-11} N^{-12}}\right)^{30 / 53}\left(\sqrt[8]{x^{1-\theta}\left(M^{11} N^{12}\right)^{8 / 23}}\right)^{23 / 53} \\
= & x^{(111-23 \theta) / 424}<x^{\theta} ;
\end{aligned}
$$

$$
\begin{aligned}
R_{9}(M, N) & =\min \left(\sqrt[5]{x^{2} M^{-2} N^{-3}}, \sqrt[8]{x^{1-\theta} M^{2} N^{6}}\right) \\
& \ll\left(\sqrt[5]{x^{2} M^{-2} N^{-3}}\right)^{1 / 2}\left(\sqrt[8]{x^{1-\theta}\left(M^{2} N^{3}\right)^{8 / 5}}\right)^{1 / 2} \\
& =x^{(21-5 \theta) / 80}<x^{\theta}
\end{aligned}
$$

(54) $\quad R_{10}(M, N)$

$$
\begin{aligned}
& =\min \left(\sqrt[30]{x^{11} M^{-11} N^{-12}}, \sqrt[32]{x^{5-2 \theta} M^{6} N^{16}}\right) \\
& \ll\left(\sqrt[30]{x^{11} M^{-11} N^{-12}}\right)^{165 / 349}\left(\sqrt[32]{x^{5-2 \theta}\left(M^{11} N^{12}\right)^{22 / 23}}\right)^{184 / 349} \\
& =x^{(357-46 \theta) / 1396}<x^{\theta} ;
\end{aligned}
$$

$$
\begin{aligned}
R_{11}(M, N) & =\min \left(\sqrt[5]{x^{2} M^{-2} N^{-3}}, \sqrt[32]{x^{5-2 \theta} M^{6} N^{16}}\right) \\
& \ll\left(\sqrt[5]{x^{2} M^{-2} N^{-3}}\right)^{22 / 54}\left(\sqrt[32]{x^{5-2 \theta}\left(M^{2} N^{3}\right)^{22 / 5}}\right)^{32 / 54} \\
& =x^{(69-10 \theta) / 270}<x^{\theta}
\end{aligned}
$$

(56) $R_{12}(M, N)=\min \left(\sqrt[30]{x^{11} M^{-11} N^{-12}}, \sqrt[32]{x^{4} M^{9} N^{16}}\right)$

$$
\begin{aligned}
& \ll\left(\left(\sqrt[30]{x^{11} M^{-11} N^{-12}}\right)^{750}\left(\sqrt[32]{x^{4}\left(M^{11} N^{12}\right)^{25 / 23}}\right)^{736}\right)^{1 / 1486} \\
& =x^{367 / 1486}
\end{aligned}
$$




$$
\begin{aligned}
R_{13}(M, N) & =\min \left(\sqrt[5]{x^{2} M^{-2} N^{-3}}, \sqrt[32]{x^{4} M^{9} N^{16}}\right) \\
& \ll\left(\left(\sqrt[5]{x^{2} M^{-2} N^{-3}}\right)^{25}\left(\sqrt[32]{x^{4}\left(M^{2} N^{3}\right)^{5}}\right)^{32}\right)^{1 / 57}=x^{14 / 57} \\
R_{14}(M, N) & =\min \left(\sqrt[30]{x^{11} M^{-11} N^{-12}}, \sqrt[26]{x^{4} M^{6} N^{10}}\right) \\
& \ll\left(\left(\sqrt[30]{x^{11} M^{-11} N^{-12}}\right)^{240}\left(\sqrt[13]{x^{2}\left(M^{11} N^{12}\right)^{8 / 23}}\right)^{299}\right)^{1 / 539} \\
& =x^{134 / 539}
\end{aligned}
$$

(59) $R_{15}(M, N)=\min \left(\sqrt[5]{x^{2} M^{-2} N^{-3}}, \sqrt[13]{x^{2} M^{3} N^{5}}\right)$

$$
\ll\left(\left(\sqrt[5]{x^{2} M^{-2} N^{-3}}\right)^{40}\left(\sqrt[13]{x^{2}\left(M^{2} N^{3}\right)^{8 / 5}}\right)^{65}\right)^{1 / 105}=x^{26 / 105}
$$

From (50) to (59), we have

$$
x^{-\varepsilon} S_{1,2,3}(M, N) \ll x^{\theta} .
$$

Lemma B follows from (48) and (60).

5. Concluding remarks. It is clear that our result 50/199 is closely connected with the term $Q^{8 / 3}$ in Theorem 2 . This term actually comes from the method given in Lemmas 3 and 4 of [1]. The fraction 50/199 can be reduced whenever $Q^{8 / 3}$ can be reduced in our Theorem 2. If, for example, $Q^{8 / 3}$ could be "omitted", then one may attain the expected exponent $1 / 4$, in place of 50/199.

Acknowledgments. This work was done during my stay in the United States. I am grateful to Mathematics Department of Columbia University for the Mathematics Scholarship financial aid, and to P. X. Gallagher, D. Goldfeld, H. Jacquet, L. Szpiro, and the participants of the Number Theory Seminar of Goldfeld \& Szpiro for stimulating conversations.

\section{References}

[1] E. Fouvry and H. Iwaniec, Exponential sums with monomials, J. Number Theory 33 (1989), 311-333.

[2] H.-Q. Liu, On the number of abelian groups of a given order, Acta Arith. 59 (1991), 261-277.

206-10, BAO GUO ST.

HARBIN, 150066

P.R. CHINA 